\title{
Was soll Plastische Chirurgie?
}

\author{
What does Plastic Surgery?
}

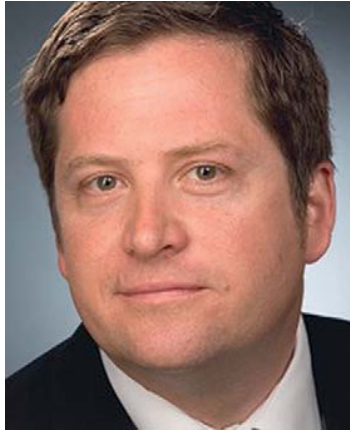

Riccardo E. Giunta

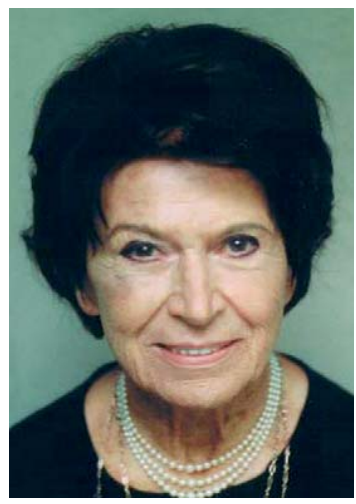

Ursula Schmidt-Tintemann

Bibliografie

DOI http://dx.doi.org/

10.1055/s-0033-1354359

Handchir Mikrochir Plast Chir

2013; 45: 191-192

c) Georg Thieme Verlag KG

Stuttgart · New York

ISSN 0722-1819

Korrespondenzadresse Univ.-Prof. Dr. med. Riccardo

\section{E. Giunta}

Handchirurgie Plastische Chirurgie und Ästhetische Chirurgie

Klinikum der Ludwig-Maximilians Universität München

Pettenkoferstraße 8a 80336 München

R.Giunta@med.uni-muenchen.de

\section{Prof. Ursula Schmidt- \\ Tintemann}

Möschenfelder Straße 66

85591 Vaterstetten
Liebe Leserinnen und Leser,

Was sie nicht soll, versteht sich von selbst: Sie darf, auch wenn es "nur“ um die äußere Erscheinung geht, nicht zum Kundendienst der Wellnessindustrie werden. Sie soll Patienten keine falschen Hoffnungen machen und die Risiken nicht schön färben. Sie darf nicht zulassen, dass sich die Indikation kommerziellen Interessen des Operateurs beugt. Kurz: Sie muss ärztliches Handeln bleiben.

Plastische Chirurgen sind Anwälte ihrer Patienten, denn nur sie können ihnen helfen, dem Druck einer aus den Fugen geratenen Operationsreklame zu widerstehen. Nur sie können sinnvolle Eingriffe empfehlen oder von kontraindizierten abraten. Nur sie können Erfolg und Risiken realistisch einschätzen.

„Mit dem Begriff der plastischen Chirurgie verbindet der Laie gewöhnlich, die Vorstellung einer Schönheitsoperation, für die eine Notwendigkeit eigentlich nicht besteht" [1].

Dieses Zitat aus der Tagespresse von 1970 zum Anlass eines der ersten Symposien für Plastische Chirurgie in München unter der Leitung der Koautorin dieses Beitrags, Frau Prof. Schmidt-Tintemann, fasst anschaulich das zentrale Problem der Wahrnehmung der Plastischen Chirurgie in der Öffentlichkeit und leider oft auch unter der ärztlichen Kollegenschaft zusammen. Schon damals wurde erkannt wie „revisionsbedürftig diese weitverbreitete Ansicht ist“.

Sind wir nach mehr als 40 Jahren weiter gekommen oder haben wir eigentlich noch das gleiche Problem? Hat es sich vielleicht sogar verschärft, weil einige dieses Bild der Plastischen Chirurgie sogar befördern?

Das vorliegende - aus zwei zeitlich unterschiedlichen Blickwinkeln- gemeinsam verfasste Editorial soll zur Diskussion über die Identität und Zukunft des chirurgischen Fachgebiets Plastische Chirurgie anregen:

Das Bild der Plastischen Chirurgie in der Öffentlichkeit - und damit in der Vorstellung potenzieller Patienten - hat gelitten. Neben vielen notwendigen Eingriffen, die die Plastische Chirurgie anbietet, gibt es auch einen Bereich, in dem es um die bereits in den 60er Jahren des letzten Jahrhunderts als wichtig erkannte „Funktion der äußeren Erscheinung“ geht [2]. Der Verdacht ist nicht von der Hand zu weisen, dass in diesem Be- reich die Indikation nicht immer das Gewicht hat, das ihr zukommt.

Es trifft sicher zu, dass die Plastische Chirurgie „den Patienten in einem bisher unbekannten $\mathrm{Maß}$ in ärztliche Entscheidungen mit einbezieht", so der Psychiater Alexander Mitscherlich zu Anlass des bereits angesprochenen Jahreskongresses der „Vereinigung der Deutschen Plastischen Chirurgen“ im Jahr 1970.

Trotzdem, oder vielleicht deswegen, reißt die kontroverse Diskussion nicht ab. Vor allem, wenn es um jene Eingriffe geht, die sich um die Funktion der Äußeren Erscheinung bemühen, um ihre Wiederherstellung nach Krankheit oder Verletzung, ihre Korrektur, etwa bei angeborenen Fehlbildungen, oder eine Verbesserung des Aussehens. Dazu der Philosoph Theodor Adorno: „You sell better, when you are a good looking person". Das Gewicht jeder Indikation wird bekanntlich von der Notwendigkeit oder nicht Notwendigkeit eines Eingriffs stark beeinflusst. Viel besser als in anderen chirurgischen Fachgebieten meint der Patient in diesem begrenzten Bereich der Plastischen Chirurgie die Ratsamkeit eines Eingriffs selber am besten beurteilen zu können. Zumal ihm das auch noch eine als Aufklärung getarnte Reklame in Print- und elektronischen Medien einredet.

Nomenklatur und Terminologie spielen also in dem Bild, das sich Öffentlichkeit und damit auch unsere Patienten von der Plastischen Chirurgie machen, eine große Rolle. Das Unwort von der „Schönheitschirurgie“, das nicht nur Laien für den Inbegriff Plastischer Chirurgie halten, verschleiert, was Plastische Chirurgie sonst noch kann und was sie vor allem tut. Von der plastisch-chirurgischen Rekonstruktion nach schweren Verbrennungen, großen Tumorresektionen oder Weichteildefekten nach Trauma bis hin zur Nervenverlagerung bei Armplexus- oder Gesichtslähmungen.

Eine Hilfsbezeichnung wie „kosmetische Chirurgie" ist ein untauglicher Versuch, dem abzuhelfen. „Kosmetisch“ suggeriert eine Beiläufigkeit und Reversibilität, die nicht zutrifft.

Erfreulicherweise wurden in den 70er- und 80erJahren in Deutschland Kliniken und Abteilungen für Plastische Chirurgie eingerichtet. Ein altes chirurgisches Fachgebiet hatte einen neuen Anlauf genommen. Die erste Generation Plastischer Chirurgen fand neue Erfahrungen und eine Weiterbildung im fortschrittlichen Ausland. Später brachte die Mikrochirurgie über die Replantations- 
chirurgie und den freien Lappenplastiken beeindruckende Erfolge in allen Körperregionen.

In der Weiterbildungsordnung 1993 erlangte die Plastische Chirurgie vor mittlerweile mehr als 20 Jahren endlich den Status eines eigenständigen, chirurgischen Fachgebiets. Sie ist samt akademischer Lehre und Grundlagenforschung zu einem wichtigen Teil der Chirurgie geworden. Die DGPRÄC hat über tausend Mitglieder. Zum Vergleich, die Deutsche Gesellschaft für Chirurgie über 6600 Mitgliedern [3].

Mit unseren starken Inhalten können wir als Plastische Chirurgen leicht aufzeigen, dass unser Fachgebiet auf Augenhöhe mit anderen chirurgischen Fachgebieten steht. Im Gebäude einer modernen Chirurgie, bei der immer mehr auch der Faktor Lebensqualität eine Rolle spielt wird die Plastische Chirurgie unvermeidlich in Zukunft noch weiter an Bedeutung gewinnen.

Trotzdem wird Plastische Chirurgie zu oft und in zu vielen Kliniken nur als Anhängsel anderer Fachgebiete, also quasi „nebenher" praktiziert. Universitätskliniken, die eigentlich eine Vorreiterrolle übernehmen sollten, sind keine Ausnahme. Zwar entstehen neue Einheiten für Plastische Chirurgie, aber andere werden geschlossen. Es zeigt sich, dass die Plastische Chirurgie nicht nur von den Chirurgen abhängt, die sie praktizieren oder organisieren, sondern auch davon, ob es für sie an einer Klinik eine dauerhafte Perspektive gibt. Kompetente Plastische Chirurgen wandern ab und machen sich selbstständig. Hochwertige Mikrochirurgie, früher großen Kliniken vorbehalten, wird zunehmend in privaten Praxen betrieben. Die Frage ist, ob selbst gehobene Positionen in der Plastischen Chirurgie noch attraktiv genug sind? Viele Plastische Chirurgen gehen lieber hinaus in den umkämpften Markt, um den notwendigen gestalterischen Freiraum zu erlangen. Das führt dazu, dass qualifizierte rekonstruktive Mikrochirurgie immer öfter in der freien Praxis betrieben und Plastische Chirurgie für Einzelfälle „Call by Call“ von anderen Fachgebieten zugekauft wird. Ein dürftiger Ersatz für fehlende Klinikeinheiten und ein Manko für die Ausbildung und Förderung von akademischem Nachwuchs.

Der Konvent der universitären Plastischen Chirurgen der DGPRÄC hat unter der Leitung von Raymund Horch in dieser Ausgabe wichtige Strategien zur Förderung des wissenschaftlichen Nachwuchses zusammen gefasst [4].

Schon immer hat die Plastische Chirurgie ihre Identität auch gegenüber anderen Fachgebieten verteidigen müssen. Unsere chirurgische Spezialität, deren erste Operationslehre vor über 400 Jahren von dem Bologneser Chirurgen Gaspare Tagliacozzi [5] niedergeschrieben wurde, hat nicht zuletzt durch die grauenvollen Verletzungen beider Weltkriege in der Moderne des letzten Jahrhunderts eine Renaissance erlebt. Als man sah, was sie leisten konnte, gab es Viele, die das Fell des Bären teilen wollten. Aber nicht Wenigen waren die Voraussetzungen dafür einfach zu mühsam: nämlich eine gründliche Ausbildung in Allgemeinchirurgie und eine mittlerweile mindestens 4-jährige, gründliche Weiterbildung in Plastischer Chirurgie.
Die Ästhetische Chirurgie ist auf einer soliden wissenschaftlichen Grundlage längst fester Bestandteil der Plastischen Chirurgie. In ihrem Marketing aber spricht sie nicht immer die Sprache ärztlichen Handelns, sondern die einer unverfrorenen Reklame, die es mit der Wahrheit nicht so genau nimmt und weder die eindruckvollen realen Möglichkeiten, noch die Grenzen der Plastischen Chirurgie ehrlich transportiert. Hinzu kommt: Die weibliche Brust ist für Print- und elektronische Medien nun einmal „good copy“. Der Erhalt einer Extremität mit einer freien Lappenplastik, die einem Patienten die Arbeitsfähigkeit zurück gibt, ist es meist nicht. Das aber ist nicht unser Problem, sondern das der Medien. Wir sollten allerdings medialer Desinformation nicht auch noch Vorschub leisten, indem wir eine falsche, von der Ausbildung her nichtssagende, und vom chirurgischen Können her bedeutungslose Berufsbezeichnung wie „Schönheitschirurgie“ verwenden. Aufklärung nach außen durch die DGPRÄC ist also gefragt. Aber auch nach innen.

Auch Auswüchse wie Bewerbung der Ästhetischen Chirurgie durch Plastische Chirurgen über Ebay ${ }^{\circledR}$, Groupon ${ }^{\circledR}$ oder anderen Plattformen degradieren den Plastischen Chirurgen zu einem Dienstleister und unterstreichen die Notwendigkeit eines Ehrenkodex.

Trotz jahrzehntelangen Bemühungen einer ganzen Fachgesellschaft kämpft der Plastische Chirurg auch in der ärztlichen Kollegenschaft noch gegen das Bild des „lifestyle-Chirurgen“. Aus der Sicht anderer chirurgischer Fachgebiete, die sich vorwiegend mit lebensbedrohlichen Tumorerkrankungen mag dieses Bild der Plastischen Chirurgie in gewisser Weise sogar nachvollziehbar sein. Tatsächlich ist es aber natürlich für den Patienten ein Unterschied, ob er bspw. mit oder ohne Extremität eine Tumorerkrankung oder eine schwere Verletzung überlebt. Die Bedeutung der Lebensqualität gegenüber dem puren Überleben von schweren Erkrankungen und Verletzungen gewinnt immer mehr an Bedeutung und macht daher eine starke Plastische Chirurgie zum unabdingbaren Puzzlestück für eine insgesamt moderne und erfolgreiche Gesamtchirurgie.

Auch in diesem Zusammenhang bietet sich eine große Chance durch den nächsten Präsidenten der Deutschen Gesellschaft für Chirurgie: Peter Vogt wird der zweite Plastische Chirurg in diesem wichtigen Amt sein.

München, im August 2013

Riccardo Giunta und Ursula Schmidt-Tintemann

\section{Literatur}

1 Püllmann A. Plastische Chirurgie oder Gesellschaftskosmetik. Wolfsburger Allgemeine 2. Oktober 1970

2 Hoopes JE, Edgerton MT, Shelly W. Organic synthetics for augmentation mammaplasty: their relation to breast cancer. Plast Reconstr Surg 1967; 39: 263-270

3 http://www.dgch.de/deutsch/ueber-die-dgch/

4 Horch RE, Vogt PM, Schaller HE et al. Strategien zur Sicherung des wissenschaftlichen Nachwuchses in der Plastischen Chirurgie - Eine Standortbestimmung und Zukunftsaussichten. Handchir Mikrochir Plast Chir 2013; 45: 193-199

5 Tagliacozzi G. De Curtorum Chirurgia. 1597 